\title{
An Undergraduate Course in Military Electronic Applications, aka Electronic Warfare
}

\section{Prof. Gene L. Harding, Purdue University}

GENE L. HARDING is an associate professor of Electrical and Computer Engineering Technology at Purdue University, where he has taught for eleven years. He has three years of industrial experience with Agilent Technologies and 28 years of combined active and reserve service in the United States Air Force. 


\title{
$\underline{\text { An Undergraduate Course in Military RF Electronic Applications, }}$ aka Electronic Warfare
}

\begin{abstract}
$\underline{\text { Abstract }}$
An applications course can be a fun and interesting way to learn about an engineering discipline. The author created a course with a focus on what the military calls electronic warfare (EW): using the electromagnetic spectrum for advantage, preserving its use for friendly forces, and preventing the enemy from doing the same. During the course, his students explored several generally-applicable topics, including various types of radio frequency (RF) propagation, highfrequency printed circuit board (PCB) layout, and antenna analysis and design. They also studied several more militarily-focused topics, including different types of military systems used in EW, processing issues with EW signals, searching for RF transmissions, and locating hostile emitters.

It is an upper-division course with an associated lab, and the author took an intentionally aggressive approach to the labs, pushing the limits of the available test equipment and facilities. As might be expected, this approach resulted in several "failures" during lab experiments, but also succeeded in pinpointing a number of issues to be addressed in future offerings of the course.
\end{abstract}

The course has now been delivered twice. Although the lecture portion remained largely unchanged between the two offerings, the lab was completely overhauled. The labs were changed to focus on a theme of defending against radio-controlled improvised explosive devices (RCIEDs). The result of this change was a substantial increase in enthusiasm and engagement for the lab portion of the course.

This paper begins with an overview of the course, including lecture topics and laboratory experiments for the first offering. It then discusses the limitations in terms of test equipment and facilities, and how some of the limitations were overcome. Both the successes and failures/lessons learned are presented, along with some of the author's observations of his students, and the course assessment data. The paper concludes with recommendations for further improvements of the course, and suggestions for implementing it at other campuses.

\section{$\underline{\text { Introduction }}$}

A very well-known and tangible application of technology can provide a strong basis for motivating student interest in a course. Such a course, if based on military applications of technology, can also impart beneficial background to graduates who pursue careers in the defense industry. Moreover, many of the topics are generally applicable, so even students who never enter the defense sector can profit substantially from the experience.

Electronic warfare (EW) is an activity the military uses in virtually all military operations. EW is using the electromagnetic (EM) spectrum for advantage, preserving its use for friendly forces, and preventing the enemy from doing the same. ${ }^{1}$ The principles governing the EM spectrum are 
the same for both military and civilian applications, and many of the issues are the same. This course, Military RF Electronic Applications, is based on the premise that a study of EW can be an interesting way to learn such radio frequency (RF) fundamentals as antenna principles, RF propagation, EM interference, and signal location. Moreover, many military applications have direct counterparts in the civilian sector: radar, navigation, and communication systems are used in both sectors, and military identification-friend-or-foe (IFF) shares some modes with civilian air traffic control (ATC) beacons. Thus, study of these systems provides useful background in both arenas.

Although there are a number of electronic warfare courses available, ${ }^{2,3,4,5}$ they are generally professional development courses that last for 2-5 days, have no labs, and are tailored for military and Department of Defense contractors. The author could not find an EW course that could be used in a university setting, so he created one.

Military RF Electronic Applications is an upper-division course with an associated lab. It includes 20 lectures, three review/problem sessions, three midterm exams, a comprehensive final exam and 12 labs spread over 15 weeks. The prerequisite is Introduction to Communication Systems, which covers basic theory and techniques of sending information, including transmitters, receivers, networks, filters, and modulation techniques. The course format is two 75-minute lectures and a 2.5-hour lab each week.

This paper is organized as follows. The next section describes the lecture content, which was largely the same for both iterations of the course. Then the labs used in the first offering are covered, including successes, failures, limitations with equipment and facilities, and guidelines used to improve the labs in the second course offering, which follows. After that is a discussion of the assessment results, including student evaluations, grade results for various aspects of the course, and how it contributes to criteria set forth by the Accreditation Board for Engineering and Technology (ABET). Following the Assessment section is a discussion of key issues for implementing the course at other institutions. Finally, the conclusion summarizes lessons learned and suggests refinements to further improve the next offering of the course.

\section{$\underline{\text { Lecture }}$}

The lecture portion of the course is divided into three blocks: 1) antennas and RF propagation; 2) military systems and EW processing; and 3) search, emitter location, and jamming. The first block of material provides a foundation for the rest of the course as the students learn what happens between the signal output of the transmitting system and the signal input of the receiving system. The second block describes, at a high level, the functions of various systems (communication, navigation, etc.), and how their signal characteristics must be analyzed to perform initial EW processing. The final block covers the issues of EW operations, including finding signals, locating emitters, and jamming them. In addition to the Adamy text, the text used in the prerequisite course is also used in the EW course. ${ }^{6}$ Other references include various web pages and system specifications. The lecture topics and associated references are listed in Table 1. 
Table 1: Lecture Layout

\begin{tabular}{|c|c|c|c|c|c|c|c|c|}
\hline \multicolumn{3}{|c|}{$\begin{array}{c}\text { Block 1: } \\
\text { Antennas \& RF } \\
\text { Propagation }\end{array}$} & \multicolumn{3}{|c|}{ 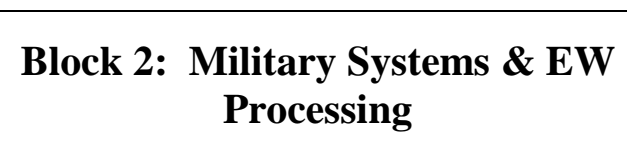 } & \multicolumn{3}{|c|}{$\begin{array}{l}\text { Block 3: Search, Emitter } \\
\text { Location, and Jamming }\end{array}$} \\
\hline \# & Title & Refs & \# & Title & Refs & $\#$ & Title & Refs \\
\hline 1 & $\begin{array}{l}\text { Course } \\
\text { Intro }\end{array}$ & Adamy $^{1}$ & 6 & $\begin{array}{l}\text { Overview, } \\
\text { Navigation }\end{array}$ & Kayton $^{7}$ & 14 & $\begin{array}{l}\text { Search } \\
\text { Issues \& } \\
\text { Strategy }\end{array}$ & Adamy $^{1}$ \\
\hline 2 & $\begin{array}{l}\text { Antennas } \\
\text { Part } 1\end{array}$ & Beasley $^{6}$ & 7 & $\begin{array}{l}\text { Communications, } \\
\text { Air Traffic Ctrl }\end{array}$ & $\begin{array}{l}\text { Beasley, }{ }^{6} \\
\text { Adamy, } \\
\text { ACP } 160 D^{8}\end{array}$ & 15 & $\begin{array}{l}\text { Search } \\
\text { Examples }\end{array}$ & Adamy $^{1}$ \\
\hline 3 & $\begin{array}{l}\text { Antennas } \\
\text { Part } 2\end{array}$ & $\begin{array}{l}\text { Beasley, } \\
\text { Adamy }\end{array}$ & 8 & Radar Part 1 & Beasley $^{6}$ & 16 & $\begin{array}{l}\text { Emitter } \\
\text { Location } \\
\text { Overview }\end{array}$ & Adamy $^{1}$ \\
\hline 4 & $\begin{array}{l}\text { RF } \\
\text { Propagation } \\
\text { Part 1 }\end{array}$ & Beasley $^{6}$ & 9 & Radar Part 2 & Beasley $^{6}$ & 17 & $\begin{array}{l}\text { Emitter } \\
\text { Location } \\
\text { Techniques }\end{array}$ & Adamy $^{1}$ \\
\hline 5 & $\begin{array}{l}\text { RF } \\
\text { Propagation } \\
\text { Part } 2\end{array}$ & Beasley $^{6}$ & 10 & Radar Ranging & Adamy $^{1}$ & 18 & $\begin{array}{l}\text { Jamming } \\
\text { Part } 1\end{array}$ & Adamy $^{1}$ \\
\hline & & & 11 & $\begin{array}{l}\text { EW Processing } \\
\text { Part } 1\end{array}$ & Adamy $^{1}$ & 19 & $\begin{array}{l}\text { Jamming } \\
\text { Part } 2\end{array}$ & Adamy $^{1}$ \\
\hline & & & 12 & $\begin{array}{l}\text { EW Processing } \\
\text { Part } 2\end{array}$ & Adamy $^{1}$ & 20 & Decoys & Adamy $^{1}$ \\
\hline & & & 13 & $\begin{array}{l}\text { EW Processing } \\
\text { Part } 3\end{array}$ & Adamy $^{1}$ & & & \\
\hline
\end{tabular}

Block one begins with a course overview, followed by an overview of EW. Electronic warfare is defined, as are its three constituents: electronic attack, electronic protection, and electronic support. ${ }^{1}$ Signals intelligence is also defined to distinguish it from electronic support. ${ }^{1}$ Other topics covered are the frequency spectrum, conversions between wavelength and frequency, and power calculations. The latter topic is covered in the prerequisite course, so this is mostly review, but does include some new additions, such as $\mathrm{dBi}, \mathrm{dBd}$, and $\mathrm{dBsm}$.

The antennas section begins with the basics needed to understand antenna operation: electromagnetic (EM) waves and polarization, reciprocity, radiation patterns and associated terms, near field vs. far field, and calculating received power. ${ }^{6}$ Dipole and monopole antennas are studied next, followed by a brief look at a variety of other antennas and antennas systems: helix, biconical, spiral, horn, loop, dish, antenna arrays, and radomes. ${ }^{1}$

The next topic is RF propagation. The concept of an EM wave is expanded to include a wavefront, plane wave, power density, electric field strength, permeability, and permittivity. ${ }^{6}$ Then wave propagation in "other than free" space is covered: reflection, refraction, and diffraction. ${ }^{6}$ This is followed by ground waves, space waves, tropospheric scatter, and sky 
waves; ${ }^{6}$ and finally, free space propagation, two-ray propagation, knife-edge diffraction, and appropriate calculations for signal strength and Fresnel zone distance. ${ }^{1}$ In addition to homework assignments, these concepts are further explored in the RF Propagation lab. This block of material culminates in the first mid-course exam.

In the Military Systems and EW Processing block, systems are divided into four primary functions: navigation, communication, identification, and location. The first category addressed is navigation systems. ${ }^{7}$ Although other types of navigation systems are briefly mentioned, the focus of this section is radio navigation systems because they are susceptible to EW. Perhaps the most vulnerable is the Global Positioning System (GPS), due to the weakness of its signal at the earth's surface. Other radio navigation systems described include LORAN, TACAN, VORTAC, ILS, and RSBN. Any of these systems can theoretically be jammed since they use RF signals. There are other possible electronic attack techniques, but they are beyond the scope of this course.

The next lecture covers communication, air traffic control (ATC), and identification-friend-orfoe (IFF) systems. Since communication systems are the focus of the prerequisite course, they are covered very briefly, primarily to address military usage. For instance, there are two primary purposes for military communications: command and control, and intelligence activities. Transmitted information may be voice or data, and the techniques used include those covered in the introductory communications course. ATC and IFF systems are presented to explain their purpose and illustrate the substantial overlap in function (ATC is civilian, IFF is military). ${ }^{8}$

The major emphasis in the systems material is on radar, which is used extensively in EW operations and is perhaps least familiar to the students. The introduction includes radar's purpose, antenna type(s), continuous wave (CW) vs. pulsed, and monostatic vs. bistatic. ${ }^{6,1,9}$ This topic was initially planned for two lectures, but was stretched to three lectures because the students struggled with the material. After the intro, pulsed and CW radars are treated separately, including range calculations for pulsed radar and Doppler shift for CW radars. Finally, radar ranging is covered. This starts with the radar range equation and its $\mathrm{dB}$ equivalent, and finishes with examples of calculating detection range (max distance the radar can detect a given target) and "detectability" range (max distance an EW receiver can detect the radar). ${ }^{1}$

The last section of material in block two is EW Processing. ${ }^{1}$ Today's signal environment is very dense and complex, and the pace of operations can be very fast. In years past EW systems often required substantial human intervention, but today the systems must be largely automated to respond in a timely manner. There is very little time to determine whether an emitter belongs to a friend or enemy, what type of system it is (comm, nav, radar, etc.), where it is, and cue appropriate systems to deal with it. For instance, if a fighter aircraft is targeted by a surface-toair missile (SAM), the entire chain of events must happen fast enough to deploy appropriate countermeasures before the fighter aircraft is shot down. Parameters to be analyzed include frequency, scan type, modulation, pulse width, pulse repetition interval, etc. The second midterm exam is administered at this point.

Block three of the lecture continues with EW operations, beginning with search strategies and issues. ${ }^{1}$ The foundational material from earlier in the course is very useful at this point. The 
topic of search is addressed first in the spatial dimension, where antenna directivity, beam width, scan rate, and receiver sensitivity are critical determinants of effectiveness. Next, search is discussed in the frequency dimension, where bandwidth, search time, and receiver sensitivity are critical. This is followed by examples used to explore the various trade-offs involved.

Emitter location is covered next. ${ }^{1,10,11}$ Several approaches to direction finding (DF) are discussed, along with emitter location techniques using DF equipment only, distance measuring equipment (DME) only, DF and DME equipment, multiple locations, and a single location. Sources of error and relative accuracy are included.

The last topic covered is jamming. ${ }^{1,11,10,9}$ There are two broad categories of jamming. Cover jamming simply overpowers the target receiver, often with a noise signal, to disrupt reception of the intended signal. Deception jamming is more sophisticated; it generally imitates a legitimate signal to trick the receiver so that it misinterprets distance, angle, or some other calculated parameter. The much-anticipated jamming lab uses cover jamming (more on that later). A third midterm exam covers the block three material, followed by a course review session and comprehensive final exam.

\section{$\underline{\text { Lab, Initial Offering }}$}

The plan for the labs in the initial course offering was to begin by designing transmitters and receivers for three frequency bands, then laying out their printed circuit boards (PCBs). The frequency bands were: garage door openers (300-400 MHz), ${ }^{12}$ Family Radio Service (462-468 MHz), ${ }^{13,14}$ and GPS (1575 MHz). ${ }^{15}$ Three weeks was allotted for these activities, after which the PCBs were ordered. Students were divided into multiple teams. Some were tasked with designing transmitters to be used for jamming; others with designing receivers for emitter location. The transmitters were to have variable output levels, and the receivers were to have indicators to show received power level. Once the PCBs were ordered, the focus shifted to antennas.

The goal of the fourth lab was to design three different antennas to be used at each frequency: a quarter-length monopole, a circular loop, and a three-element Yagi-Uda array. Construction was relatively crude, using 16-gauge solid copper wire, cardboard, a hot-glue gun, and SMA cables. Students were required to perform pre-lab calculations to determine physical dimensions for constructing each antenna.

The next lab measured radiation patterns of the various antennas, taking a measurement every $30^{\circ}$ in azimuth $\left(0^{\circ}\right.$ elevation). Unfortunately, we did not have access to an anechoic chamber, which would have been ideal, and did not have a portable spectrum analyzer, so going outside was not an option. In retrospect, it might have been interesting to use a large auditorium (if available), but the author did not think about it at the time. The cluttered environment of the lab was not a good place to do pattern measurements, and the results were poor. There were two other big problems with this lab: there was no commercial (i.e., tested) antenna to use as a baseline for each test, and nine antennas was too many. One set of antennas (monopole, loop, and Yagi-Uda) at a single frequency would have been sufficient, and made the lab activity much 
more manageable. Moreover, a commercial antenna would have provided a useful baseline for comparison.

Two weeks were spent studying RF propagation. The lack of access to either an anechoic chamber or a portable spectrum analyzer again limited activity to the indoor lab. Results were probably OK, given that maximum transmitter-receiver separation was only about 7 meters, but the change to outdoor measurements in the second iteration of the course was a substantial improvement.

Two labs were set aside to assemble and characterize the transmitters and receivers, which turned out to be a disappointment. Even the top students in the class never got their systems working. Thus, the live labs to perform search and emitter location were canceled, as were the jamming labs.

As an alternative, a MATLAB-based lab was developed to simulate a scan-on-scan search scenario with two circularly scanning narrow-beam antennas. The objective was to estimate the probability of intercept (POI) and to compare it to theoretical values. The problem was set up to assume that the search receiver could only see the emitter when the two antennas were pointing at each other (i.e., within the 3-dB beam width of each antenna). Many of the students struggled with the MATLAB code, and no one achieved good correlation between the theoretical and simulated values. It was an interesting exercise in probability calculation and simulation, but the scenario and simulation need to be scrutinized more thoroughly if used again.

An alternative was also developed to perform jamming. The author had a crude, short-range GPS jammer that he had built for a different project several years before, and three of the students had GPS units in their cars; units that could be removed and run on their own batteries' power. An intramural flag football/soccer field was chosen as a test site, and experiments run there. Unfortunately, two of the students discovered that their GPS units' batteries died very quickly after being removed from their cars, so we were only able to test with one GPS unit. The tests were, however, reasonably successful. An additional impromptu test was executed on a footbridge over the river. This footbridge had a very substantial steel handrail on each side, which appeared to create a fading situation. Unfortunately, the third unit died shortly after these tests began, so results were inconclusive. The potential fading situation is quite intriguing, and worth further investigation.

The lab portion of this first offering of the course had a number of problems. As mentioned above, lack of either an anechoic chamber or a portable spectrum analyzer made "free space" RF measurements very problematic. Additionally, although there were three spectrum analyzers in the lab, two of them were unreliable and were eventually removed from the lab. The lack of equipment really limited what measurements could be performed.

In retrospect, the goals for the labs were overly ambitious. The labs tried to accomplish too many disparate aims, and very little was accomplished effectively. More focus was needed. Recommendations after the first course offering were to limit the frequencies to one band only, one antenna type only, and one type of EW function. The second offering should be more focused, and try to do a few things well. Variety could be added later, if appropriate. 


\section{$\underline{\text { Lab, Second Offering }}$}

The lab portion of the second iteration of the course was completely overhauled. A theme was chosen to provide meaning and focus for the semester's lab activities. They would be based on a very tangible and well-known scenario: a radio-controlled improvised explosive device (IED). This turned out to be a good choice. Although only two of the students had prior military service, an informal poll revealed that everyone had a family member or friend who was serving or had served.

Since garage door openers are plentiful, and used ones were assumed to be readily available, that became the basis for the labs. The author contacted a local garage door opener installer (Overhead Door ${ }^{16}$ ) and asked if they would save some old openers when they removed them, if they still had working radios. The owner agreed, and secured a few openers for the course.

At a high level, the lab sequence was:

1. Analyze and disassemble each opener to figure out how the radio portion worked.

2. Determine what was needed to turn the opener radios into mock IEDs, and complete the design.

3. Design jammers to defeat openers across the entire band.

4. Lay out and order PCBs for the jammer circuits.

5. Design and test a monopole antenna for the jammer.

6. Build and bench test the mock IED, antenna, and jammer.

7. Perform an RF propagation lab (outside) to get a better understanding of the propagation issues involved for the jamming scenario.

8. Write a test plan for the jamming lab (rather than the instructor providing one).

9. Execute the jamming lab (outside) to test effectiveness of each jammer.

The first lab was called a Foreign Materiel Exploitation, which is the term the U.S. military uses to describe the detailed analysis of a foreign system for the purpose of learning how to exploit and/or defeat it. This lab had two objectives:

1. Identify key parameters needed to convert the radio receiver of a garage door opener ("operator") to battery power.

2. Extract the radio receiver from the operator and demonstrate its operation with DC power on the lab bench.

The students were immediately engaged in this lab, seemed to thoroughly enjoy it, and everyone achieved the goals before lab time expired. Although lab three was Mock IED Design, the plan is to incorporate this exercise into lab one next time. This can be done with an additional goal for a post-lab analysis: Design the mock IED, select components, and order them.

The second lab was the jammer transmitter design. It had a single goal: Design a variable-gain transmitter to operate across the garage door opener frequency band(s) and select the components. Although generally successful, a few changes could improve this lab next time. The following topics could be covered at the end of the first lab, in a pre-lab assignment for the second lab, and/or in an early lecture: 
1. The concept of frequency spreading, so students understand what must be done to cover the entire frequency band $(300-400 \mathrm{MHz})$;

2. The voltage-controlled oscillator (VCO, and how it can be used for frequency spreading;

3. The zero-and-span amplifier, as a possible mechanism to drive the VCO.

The fourth lab was the jammer circuit PCB layout. The goal was simple: lay out a PCB for the transmitter. While generally successful, this lab could be improved. Based on instructor observations and student feedback, it appears that this lab should be slipped a week, and a lab inserted to provide a tutorial of PCB layout software. Although a previous course (sophomore year) did involve PCB layout, it was apparent that the students did not learn the software thoroughly. All of them encountered problems setting up their boards. Recommendation: The instructor should learn this (or another) software package for layout, and provide a tutorial for the third lab. Moreover, the pre-lab for the following week should be some key part of the layout to ensure completion of the layout before lab 4 is completed.

Lab five, planned to become lab six in the next iteration of the course, was Antenna Design. Its objectives were:

1. Construct an omnidirectional antenna for the $300-400 \mathrm{MHz}$ frequency band.

2. Prepare an Excel spreadsheet to input antenna measurement information and plot the radiation pattern in rectangular and polar formats.

3. Design an impedance matching network to match the antenna to an SMA connector. Pre-lab work for this lab included reading about monopole antenna design, calculating antenna parameters, and reviewing low-pass L-network design (material covered in the prerequisite course). The lab itself involved building the antenna, creating the data collection spreadsheet, and designing the L-network. All of the students did well in this lab.

The next lab, which would become lab five in the next offering of the course, was to build and test the mock IED using the radio from the garage door opener. This lab had three objectives:

1. Finish construction of the mock IED.

2. Given a set of test objectives, write a test plan to test the mock IED.

3. Test the mock IED to verify its operation and determine its maximum operating range. Everyone did pretty well accomplishing the first objective. Writing the test plan, however, was more problematic.

Although most of the changes to the labs involved simplification and focus, there was one intentional change that increased the difficulty: development of test plans. The author was not generally happy with his observations of upperclassmen's ability to write effective test plans (a deficiency he also saw in industry), so this activity was added to a couple of the labs.

In this lab, the test objectives were provided (a key first step):

1. Ensure the operational indicator(s) (light, buzzer, etc.) work correctly. (NOTE: "Work correctly" refers to hearing the buzzer, not electrical parameters.)

2. Determine effective range at which the indicators can reasonably be detected in normal daylight conditions outside (i.e., how far the buzzer can be heard, if its range is less than that of the transmitter range).

3. Determine effective range of the remote control transmitter (i.e., how far away it works). 
4. Measure received power at the mock IED in a manner that allows comparison among the four mock IEDs and the four jammers (i.e., the distance must be the same for each transmitter power level measurement).

This was followed by a short explanation of how to divide a test plan into phases, then the following questions to address for each phase:

- What parameters need to be measured, AND their units (e.g., power level in $\mathrm{dBm}$, distance in lightyears, etc.)?

- What are the expected values (or range) for each parameter (e.g., 4.9 - 5.1 V)?

- How will the data be recorded (pen and paper, laptop PC, lab benchtop PC, etc.)?

- What equipment is required to perform the test (DMM, oscilloscope, etc.)? Remember to include model number of the equipment (e.g., Agilent N9340B handheld spectrum analyzer).

Additionally, the lab instructions advised students to think about how to effectively present the data after it was collected.

The following template was in the lab instructions to help them organize the test plan:

\section{Bench Test}

Equipment: Add equipment list here.

Step-by-step:

1. You write the instructions.

2. ...

\section{Range Tests (remember that there are two different test objectives here)}

Equipment: Add equipment list here.

Step-by-step:

1. You write the instructions.

2. ...

\section{Received Power Test}

Equipment: Add equipment list here.

Step-by-step:

1. You write the instructions.

2. ...

The big change that needs to be made to this lab is a more rigorous grading rubric. The rubric set up for this lab only required the specification of test equipment and the presence of tests for the four objectives. The rubric needs explicit checks to ensure the bullet questions are answered: parameters and their units, expected values, how the data will be recorded, and specific model numbers of the equipment. Since the original rubric did not require it, there is no hard data, but the instructor's recollection is that some of the students were not thorough in answering the first three questions.

In lab seven, students finished their antennas and tested them. These were the objectives:

1. Finish construction of the monopole antenna.

2. Learn how to use the spectrum analyzer's tracking generator.

3. Plot the antenna's frequency response. 
Pre-lab steps included completion of the antenna, some reading about the spectrum analyzer, watching a video tutorial on how to use the analyzer's tracking generator function, and writing the test plan. In this case, the author was only looking for the test plan to indicate that students had done the reading and watched the video. During lab, students used the tracking generator function to generate their antennas' frequency responses (one wideband, one narrowband). Students did well in this lab. No changes are recommended.

The class investigated RF propagation in lab eight, using free-space and two-ray propagation models. Pre-lab work included calculating Fresnel zone distance, hypothesizing about an easily reversible modification that could be made to the antenna to increase its effective radiated power (ERP), and creating and printing the data table to be used for data collection in lab. The objectives for this lab were:

1. Calculate propagation loss in various scenarios and compare to live measurements.

2. Estimate transmitter power based on live measurements.

3. Compare antenna effective radiated power (ERP) with and without a beam focusing mechanism.

The pre-lab and in-lab portions of the grades were pretty good (80\% and 100\%, respectively), but the post-lab analysis and conclusions were inconsistent. Scores ranged from $13 \%$ to $85 \%$, with an average of $41 \%$. The issue of poor grades in this case appeared to be either lack of effort or not following the directions (or both). The students who made a concerted effort did fairly well. No changes are recommended for this lab.

Lab nine, Write Jamming Lab Test Plan, was completely different from the other labs. The format was a group discussion, with each student leading a different portion of the discussion, and the instructor providing input and questions along the way. The objectives were:

1. Develop a step-by-step test plan to test $\underline{\text { each }}$ jammer against $\underline{\boldsymbol{e a c h}}$ mock IED.

2. Lead part of the discussion.

3. Participate in all of the discussion.

4. Document part of the discussion.

In this lab students developed portions of two different test plans: the lab bench test of the jammer, and the range test of the jammer against the IED. In each case they were tasked to come up with test objectives, how to meet them, parameters and units to be measured, test equipment needed, testing phases, how to record data, expected values/tolerances, data presentation method, and data needed from other teams. The students later told the instructor that this lab was very enlightening, very useful. They clearly had trouble contemplating and formulating all of the information needed for the test plans. For instance, at one point they were discussing how to characterize the jammer's output for the lab bench test. After they determined they would measure and record the power, the instructor asked how they would record it. They responded they would write it down. It took a few leading questions over a period of several minutes of discussion to get them to finally realize that they were characterizing the jammer from 300-400 $\mathrm{MHz}$ and that they either needed a series of measurements at different frequencies or a screenshot of the spectrum analyzer that included the entire frequency band. This was one of several moments during the lab that helped the class understand the thought process that goes into developing an effective test plan. It was edifying to get direct feedback from several students that they "got it" during this lab. 
In lieu of lab ten, the instructor held a schedule review designed to get feedback from the students. Feedback was generally positive, with a few minor suggestions for feedback, but one significant deficiency was identified: the need for a tutorial on PCB layout. This issue was discussed above, along with lab four, and the tutorial exercise is planned to become lab three in the next iteration of the course.

The lab to build and bench test the jammer had the following objectives:

1. Finish construction of the jammer.

2. Tune and bench test the jammer to verify its operation, measure its 3-dB bandwidth, and characterize its frequency performance in the "threat" spectrum of 300-400 MHz.

Two-thirds of the class got their jammers to work. Those who did not have functional jammers did not do well. Ironically, of the others, half had strong jammer performance but weak analysis, while the other half had poor jammer performance but strong analysis; their average grade was $74 \%$.

The climactic lab was the IED Jamming lab. All teams did well on the pre-lab (88\%), but lab performance was mixed (73\%), as was the post-lab analysis (50\%). Overall lab average was $74 \%$. The poor performance on the part of some students was primarily due to not following instructions, so no significant changes are recommended for this lab. The team with the best performing jammer suppressed the garage door opener from over 200 feet away (opener remote was 30 feet away). Also, the team that never got their jammer working participated appropriately in this lab, so they were allowed to do an alternative assignment to substitute for their grades in this lab and the previous lab. The assignment was a report comparing and contrasting the design approaches taken by the different teams.

\section{$\underline{\text { Assessment }}$}

This course has five learning objectives:

1. Describe and apply the different types of electronic warfare.

2. Analyze and design several antenna systems.

3. Analyze and predict RF propagation characteristics under various conditions.

4. Describe the function and operation of a range of military systems.

5. Apply a variety of EW techniques and characterize their effectiveness in different operating environments.

Although there were several homework assignments and quizzes during the course, the tools used for summative assessments were three midterm exams, a comprehensive final exam, and a subset of the laboratory exercises.

Objective one was evaluated using questions from exam one and the final exam, with a class average of $82.3 \%$. No changes were recommended. The second objective was assessed with both exam questions and laboratory exercises. The average for questions from exam one and the final exam was $77.1 \%$, while the average performance for the antenna labs was $85.5 \%$. No changes were indicated. 
Objective three was also assessed using both exam questions and laboratory exercises. Although the laboratory exercise results were marginally acceptable at a $74.9 \%$ average, the lab instructions have been modified for increased clarity the next time the course is offered. The exam questions' average was not acceptable: only 63.7\%. A closer review of the exam questions revealed poor scores for calculating received power, Fresnel zone distance, and propagation loss. Although the latter two improved somewhat from exam one to the final exam, the instructor plans to add more homework and/or quiz problems to give students more practice.

The fourth objective was covered by questions from exam two, exam three, and the final exam. The class average was $83.9 \%$, so no changes are indicated. Objective five was also assessed by questions from the second, third, and final exams. The scores for radar detection range and detectability range were especially poor ( $33.3 \%$ and $0 \%$, respectively), so these topics will be emphasized more in future offerings.

From a program perspective, this course contributes to ABET criteria $3 \mathrm{a}-3 \mathrm{~g}$ for engineering technology programs. ${ }^{17}$

Student feedback on the course was generally positive. They rated the lecture and lab portions of the course at 4.0 and 3.9, respectively, on a 5.0-point scale. Written comments included the following:

- The professor is very knowledgeable about the material.

- I like that the instructor always has the information available online.

- Labs were interesting, applicable, and well thought out.

- Recorded lectures make studying and review easy.

\section{$\underline{\text { Implementing at Other Institutions }}$}

The topics and sources used in this course are listed elsewhere in the paper. Interested instructors who want to implement the course at their schools are welcome to contact the author for more detailed information. This section will address a few issues of particular importance to course success.

The first issue relates to laboratory exercises. After teaching the course twice, the author believes it is very important to have spectrum analyzers in the lab. Ideally, it is good to have spectrum analyzers with built-in tracking generators to facilitate filter characterization. Using the spectrum analyzers is important for familiarizing the students with the instruments' use.

A second issue also relates to labs: a method of measuring propagation loss. Although an anechoic chamber would be nice, many schools do not have or have access to such a facility. The author found that free range measurements were an acceptable alternative. Although a large, empty parking lot was used, a grassy field would also be an acceptable location. Either way, at least one of the spectrum analyzers must be portable, i.e., battery powered, to enable testing outside. 
Finally, a third challenge is that of the text. In the first two offerings of the course, the author was able to require a single textbook, the one by Adamy. ${ }^{1}$ This was because the prerequisite course required the Beasley text. ${ }^{6}$ This will be continued the next time the course will be offered, but after that time the prerequisite course will no longer require a text, so the author faces a quandary: require two textbooks, or pick one and make the other optional. For the sake of the students' finances, the latter option might be more preferable. If forced to choose one of the two, the Beasley text is probably better because it contains more generally applicable information (i.e., topics applicable to both civilian and military situations). On the other hand, used textbooks can now be acquired easily online, usually at reasonable prices, so it may be acceptable to require two textbooks.

\section{$\underline{\text { Conclusion }}$}

The subject course, Military RF Electronic Applications, has been offered twice. Results, especially with regard to the second offering, indicate good success. The average course grade was $83 \%$, student engagement was high, and two-thirds of the class succeeded in creating a successful jammer. Student feedback was generally positive for the second offering: $4.0 / 5.0$ for the lecture, 3.9/5.0 for the lab. These scores were up substantially from 2.5/5.0 and 2.5/5.0 for the lecture and lab portions of the initial offering.

The big difference between the two course offerings was the lab portion. Most of the changes were based on a philosophy of focus and simplicity. These changes included focusing design and testing on a single frequency band and using only one antenna type. The garage door frequency band was used, while the FRS and GPS frequency bands were eliminated. Likewise, the only antenna type used was the monopole. The Yagi-Uda and loop antennas were dropped.

Another change that seemed to help student engagement was the IED theme. Almost all of the students had friends or family members who were veterans (as were two of the students), so the lab tasks were very relevant and interesting to them. Some of the lab tasks required students to compose their own test plans. Although challenging for the students, based on their feedback to the instructor, most of them appreciated the benefits of those tasks.

The course should be very scalable to other interested institutions. Key facility/equipment requirements include spectrum analyzers and an ability to perform live RF propagation testing. Schools that send a substantial percentage of graduates into the defense industry may want to consider implementing this course.

\section{Bibliography}

\footnotetext{
${ }^{1}$ Adamy, D. (2001). EW101: A First Course in Electronic Warfare. Norwood, MA: Artech House.

${ }^{2}$ Stark, A. et al (2014). Fundamentals of Photonics in EW Applications. Georgia Tech Professional Education. Retrieved February 10, 2014 from http://www.pe.gatech.edu/courses/fundamentals-photonics-ew-applications.

${ }^{3}$ Adamy, D. (2014). Electronic Warfare Overview. Applied Technology Institute. February 10, 2014 from http://www.aticourses.com/electronic warfare_overview.htm.
} 
${ }^{4}$ Wiley, R.G. (2014). Introduction to Radar and Electronic Warfare, Association of Old Crows. Retrieved February 10, 2014 from http://www.crows.org/details/253-radar-and-electronic-warfare.html.

${ }^{5}$ Berg, Lynn (2014). Electromagnetic Battle Manaagement Concepts, Association of Old Crows. Retrieved February 10, 2014 from http://www.crows.org/details/260-electromagnetic-battle-management-concepts.html.

${ }^{6}$ Beasley, J.S., Miller, G.M. (2008). Modern Electronic Communication. Upper Saddle River: Pearson Education.

${ }^{7}$ Kayton, M. (2001). Navigation Systems. The Avionics Handbook. CRC Press LLC. Retrieved February 10, 2014 from http://helitavia.com/avionics/TheAvionicsHandbook_Cap_13.pdf.

${ }^{8}$ Combined Communications Electronics Board (Australia, Canada, New Zealand, United Kingdom, United States) (2007). IFF/SIF Operational Procedures ACP 160(D).

${ }^{9}$ Stimson, G.W. (1998). Introduction to Airborne Radar. Raleigh, NC: SciTech Publishing.

${ }^{10}$ Schleher, D.C. (1986). Introduction to Electronic Warfare. Norwood, MA: Artech House.

${ }^{11}$ Graham, A.W. (2011). Communications, Radar, and Electronic Warfare. West Sussex, United Kingdom: John Wiley \& Sons, Ltd.

${ }^{12}$ Garage Door Opener, https://en.wikipedia.org/wiki/Garage door_opener, retrieved February 11, 2014.

${ }^{13}$ FCC Encyclopedia: Family Radio Service, http://www.fcc.gov/encyclopedia/family-radio-service-frs, retrieved February 11, 2014.

${ }^{14}$ Family Radio Service, https://en.wikipedia.org/wiki/Family_Radio_Service, retrieved February 11, 2014.

${ }^{15}$ Global Positioning System, https://en.wikipedia.org/wiki/Gps, retrieved February 11, 2014.

${ }^{16}$ Overhead Door Corporation, http://www.overheaddoor.com/Pages/LandingPage.aspx, retrieved February 12, 2014.

${ }^{17}$ Criteria for Accrediting Engineering Technology Programs, 2012-2013, http://www.abet.org/DisplayTemplates/DocsHandbook.aspx?id=3150, retrieved February 15, 2014. 